\title{
The advantages of making science accessible
}

\author{
Erika Labbé $a$, * \\ a Núcleo de Astronomía, Universidad Diego Portales, \\ Av. Ejército Libertador 441, Santiago, Chile \\ E-mail: astrodifusion@mail.udp.cl
}

At first it was a challenge of scientific communication, now it is a way of life. Making science accessible to people with disabilities holds a potential that goes beyond social responsibility or empathy. It is also about making diversity an integral part of scientific development, incorporating people full of curiosity with new approaches to face problems. We propose to start by revising our own perception of disability, giving people with disabilities and people involved in their education and caretaking the opportunity to tell us about it, and by opening our minds to new ways of doing science. Inclusive astronomy drives us to be creative, to generate networks and to collaborate in an interdisciplinary way. We hope that this initiative will extend to other areas of scientific research. 


\section{Introduction}

Communicating astronomy without images for Blind and Visually Impaired (BVI) people was an outreach challenge we started to address at the beginning of 2016. Our first experience was about star formation, using a well-developed story and simple tactile models (Fig. 1). On that occasion, we had the help of collaborators from "Bibliociegos" (Central Library for the Blind, Santiago, Chile), the place we chose to carry out this experience. The activity was very successful for all the public (BVI and sighted), and we soon found out that we were not only adapting an astronomy talk for BVI people, but also building something new and very important, and we couldn't do it alone.

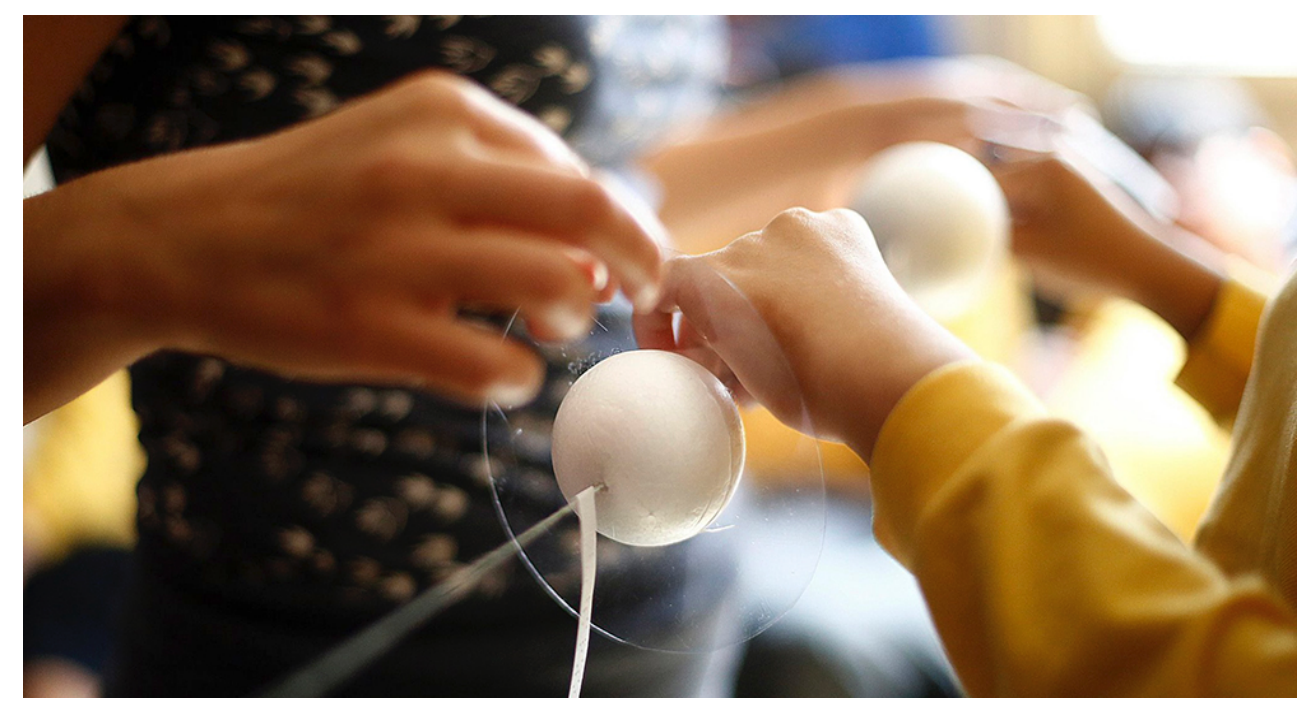

Figure 1: Solar System tactile model, being explored by a visually impaired student during the first experience.

The first decision we made was to share this new experience: training other astronomers and students, collaborating with scientific institutions with the same interests, approaching groups of BVI people to invite them to participate, and finally build a community called "Astronomía Inclusiva" (Inclusive Astronomy), in order to learn together how to make science more accessible. After 5 years, we have good reasons to invite all scientific communities to join.

\section{What is inclusive astronomy?}

When we think about the barriers of astronomy for people with disabilities, many of them seem obvious, but they are not always the correct ones. Most of the time we lack the information to make the correct assumptions. For example, if you do not know about the screen reading software that a blind person can use on their smartphone, it will be difficult to imagine how they can use it.

\footnotetext{
${ }^{1} \mathrm{https}: / /$ www.facebook.com/biblioteca.centralparaciegos

${ }^{2}$ https://www.facebook.com/astroinclusiva
} 
Some of the barriers that a person with a disability faces in astronomy and science in general are:

- Usage of visual representations.

- Lack of sign language signs for technical terms.

- Physical barriers to access labs and science buildings.

- Short time to fulfill high productivity standards.

- Prejudices.

- Overprotection.

Some of these are very practical and can be solved by working on accessibility tools, such as a data sonification software. Others are mostly attitudinal and need a change in mindset. Many affect us all, but are even more challenging for people with disabilities.

To overcome these barriers, accessibility must be addressed at all levels: Outreach, Education, and Research.

What does it mean? For naturally curious scientists, it can be a lot of fun - it's an opportunity to explore other ways of perceiving the world and increase the possibilities, instead of reducing them. Scientific research can benefit from this, for example, improving the data analysis through sound as proposed in W. Diaz-Merced's PhD thesis [1] $]^{3}$.

This exploration leads to the creation of new ways of communicating, not only in outreach settings, but also in education and research.

Observing the reactions of people, with and without disabilities, to this new way of communicating science is very rewarding and shows that inclusion actually implies an improvement in communication for everyone. There is no need to keep it only for people with disabilities.

It also means that we have to work on some aspects of scientific development, such as motivation, interdisciplinary collaborations, reasonable accommodations, and mindset changes.

\subsection{Motivation}

In our experience, some of the communities with disabilities are less motivated by astronomy than others, and we have some ideas why: visually impaired people are less exposed to astronomical images, which are very common to find in the media in a country with many astronomical observatories like Chile. Hearing impaired people are not used to have sign language interpretation on astronomy talks, and they don't look for it. But all of them are just as curious as anyone else.

\footnotetext{
${ }^{3}$ https://theses.gla.ac.uk/5804/
} 


\subsection{Interdisciplinary collaborations}

In order to change this, projects such as "Breaking the Barriers"4 of the Chilean Astronomical Society (SOCHIAS), are working to promote the use of chilean sign language interpreters in outreach events, offering financial support to all the astronomical institutions in Chile, while other institutions and projects are developing experiences with tactile models, sounds, and image descriptions of astronomical objects and phenomena aimed at different ages and educational levels (Fig. 2). Within the astronomical community, SOCHIAS created an Inclusion and Gender Working Group ${ }^{5}$ in 2019, and they are working with a team of advisers made up of people with disabilities that participate in the creation of guidelines and surveys, preparing the way for future astronomers with disabilities.

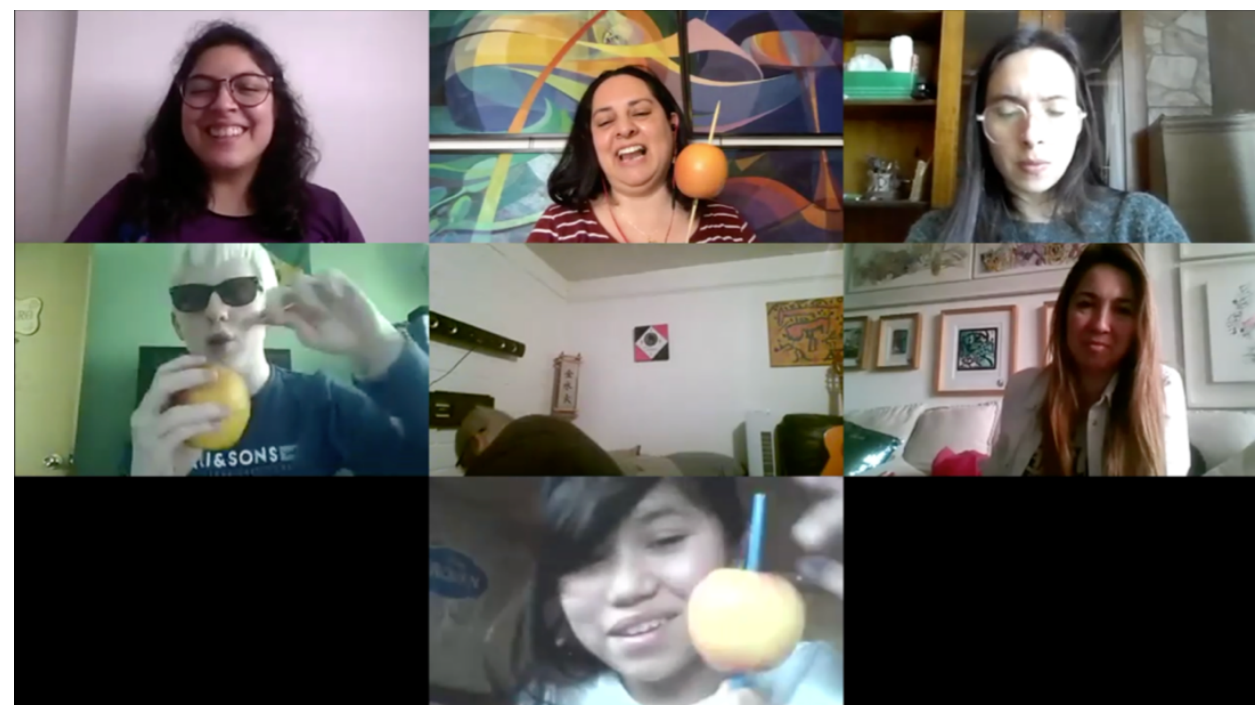

Figure 2: Santa Lucía School BVI students, teachers and scientists (Dedoscopio ${ }^{6}$ project and AstroUDP $^{7}$ ) in the process of creating an online inclusive experience about celestial movements.

This development has been possible thanks to an interdisciplinary collaboration, and the "Astronomía Inclusiva" group ${ }^{8}$ became a space that allows us to have this kind of interaction. It is currently composed of 110 members, including astronomers, people with disabilities, teachers, disability experts, parents, artists, designers, other scientists, etc., from 10 countries, many of them associated to more than 25 science institutions and projects (Fig. 3), all of them connected via WhatsApp.

\footnotetext{
${ }^{4}$ https://sochias.cl/actividades/difusion/breaking-the-barriers/

${ }^{5} \mathrm{https}$ ://sochias.cl/actividades/grupo-de-trabajo-en-inclusion-y-genero/

${ }^{6} \mathrm{https}: / / \mathrm{www}$. facebook.com/dedoscopio/

${ }^{7} \mathrm{https}: / /$ astronomia.udp.cl/

${ }^{8} \mathrm{https} / / /$ astronomia.udp.cl/es/espanol-primera-reunion-del-grupo-de-astronomia-inclusiva-se-realizo-en-la-udp/
} 


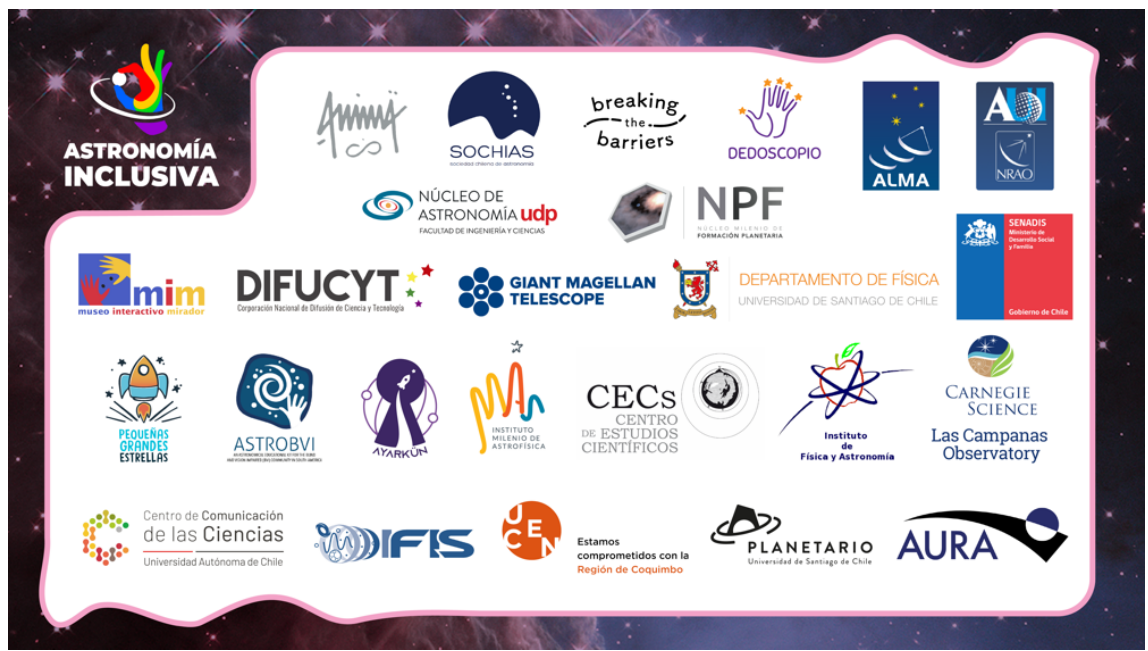

Figure 3: Scientific institutions and projects represented by members of "Astronomía Inclusiva" group.

\subsection{Reasonable Accommodations}

Defined as: "Necessary and appropriate modifications and adjustments not imposing a disproportionate or undue burden, where needed in a particular case, to ensure to persons with disabilities the enjoyment or exercise on an equal basis with others of all human rights and fundamental freedoms" $[2]^{9}$, reasonable accommodations are important at all stages of the scientific career and are expected to be defined in collaboration with people with disabilities.

As we have shown, inclusion in astronomy is not just about accessibility tools, it also requires a change of mentality.

\subsection{Some important mentality changes}

- A person with a disability knows his/her own limits better than the rest. "Common sense" is not always enough.

- A person with a disability does not seek pity, but equity, even if our first reaction is exactly that one.

- More adaptability, less perfectionism: Let's allow ourselves to fail.

\section{Diversity}

People with different ways of facing challenges bring creativity and tools to achieve better solutions. A good example: data sonification. Sonification is the use of non-speech audio to convey information or perceptualize data $[3]^{10}$.

\footnotetext{
${ }^{9} \mathrm{https} / / /$ www.un.org/development/desa/disabilities/convention-on-the-rights-of-persons-with-disabilities.html

${ }^{10} \mathrm{https}$ ://www.worldcat.org/title/auditory-display-sonification-audification-and-auditoryinterfaces/oclc/923848368
} 
The astronomer Wanda Díaz-Merced found out that "Multimodal stimulus in synchrony with the sound helps space scientists identify events masked by noise in $2 \mathrm{D}$ data" in her PhD thesis [1]. Her work, and that of other astronomers with and without visual impairment, proposes that data sonification can provide an advantage for all scientists.

\subsection{Some sonification tools}

- $\quad$ Sonify ${ }^{11}$ (NASA Space Physics Data Facility (SPDF))

- Astronify $^{12}$ (HST)

- StarSound and Vox Magellan ${ }^{13}$ (Swinburne University)

- $\operatorname{SonoUno}^{14}$ (ITeDA, EGO)

- Sonification Sandbox ${ }^{15}$ (Georgia Institute of Technology)

- $\quad$ Sonifyer ${ }^{16}$ (Berne University of the Arts (BUA))

- SoniPy $^{17}$ (David Worrall)

- Afterglow Access ${ }^{18}$ (IDATA, High school level)

Sonification is also starting to be used in outreach, as you can find in the twitter account @ASASSN_bot ${ }^{19}$ (Fig. 4), which shows sonified optical light curves of variable stars from the All-Sky Automated Survey for Supernovae ${ }^{20}$ (ASAS-SN).

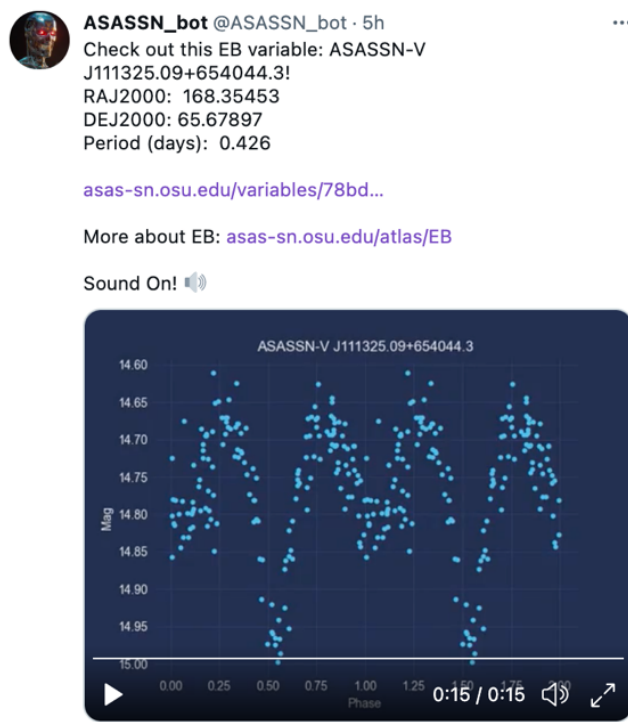

Figure 4: Sonification on @ASASSN_bot in Twitter

\footnotetext{
${ }^{11} \mathrm{https}$ //sourceforge.net/projects/xsonify/

$12 \mathrm{https}$ ://astronify.readthedocs.io/en/latest/

${ }^{13} \mathrm{https}: / /$ www.youtube.com/watch? $\mathrm{v}=\mathrm{gQdvv}-\mathrm{gCa} 3 \mathrm{Y}$

${ }^{14} \mathrm{http}: / /$ sion.frm.utn.edu.ar/sonoUno/

${ }^{15} \mathrm{http} / / /$ sonify.psych.gatech.edu/research/sonification_sandbox/

${ }^{16} \mathrm{http}: / /$ www.sonifyer.org/

${ }^{17} \mathrm{https} / / /$ www.sonification.com.au/sonipy/index.html

$18 \mathrm{https} / / /$ idataproject.org/resources/

${ }^{19} \mathrm{https}: / /$ twitter.com/ASASSN bot

${ }^{20} \mathrm{http}: / /$ www.astronomy.ohio-state.edu/ assassin/index.shtml
} 
Sharing these inclusive astronomical experiences makes the general public begin to change their expectations of people with disabilities, and it also changes the expectations of people with disabilities about themselves (Fig. 5).

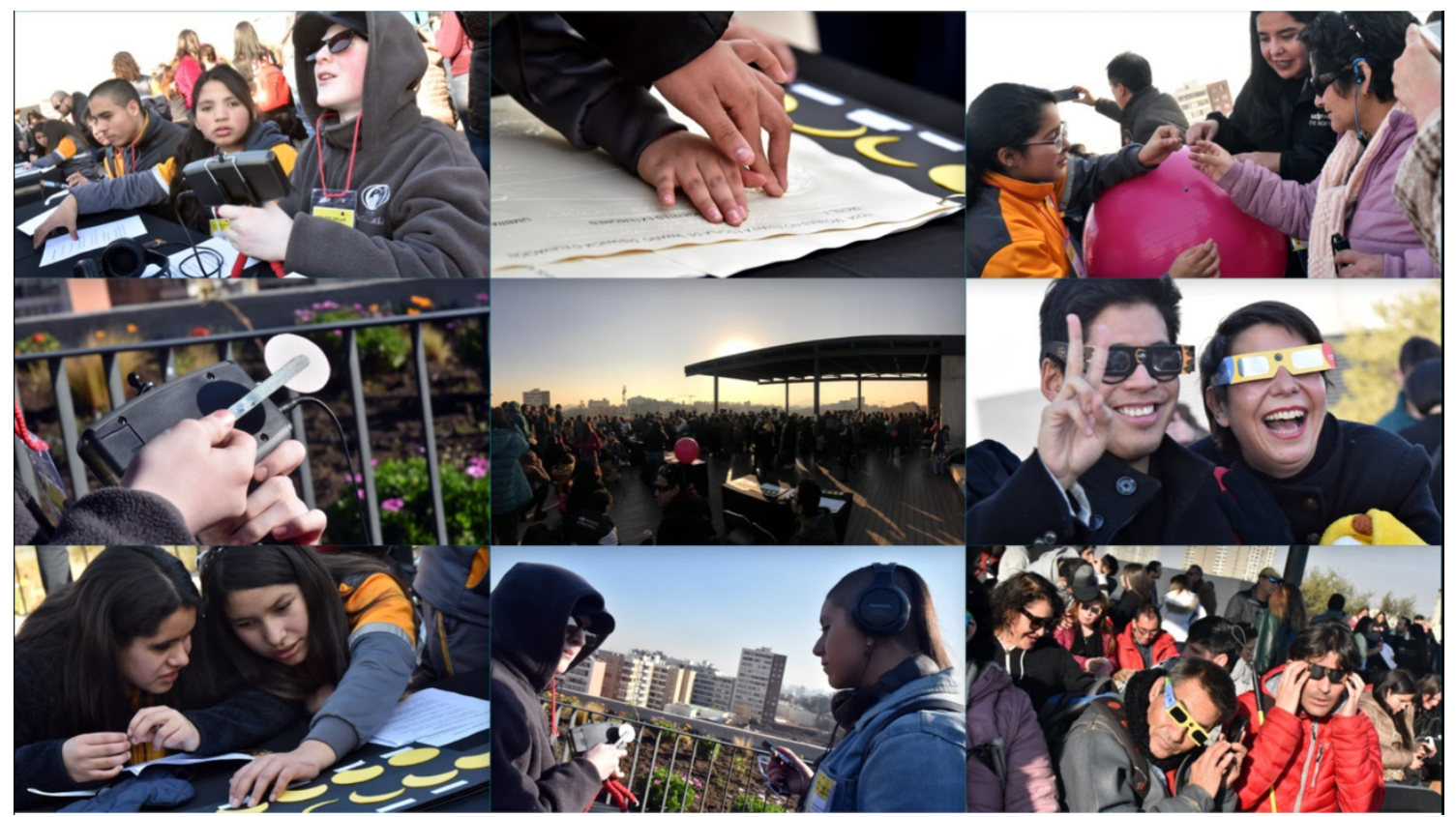

Figure 5: Santa Lucía School'1 BVI students hosting the UDP 2019 solar eclipse activities at UDP.

\section{How to start to become inclusive?}

We recommend choosing a disability to start with, and learn what it is about.

The communities of people with disabilities are usually open to helping scientists learn to be inclusive, as long as we invite them to participate in the process. That is what their motto "Nothing about us without us" stands for.

Try something simple to get started and find the potential in it.

For example: learning about image description shows that the images alone do not tell the whole picture - a galaxy is not just that beautiful patch of light and color that we see in the image. It is incredibly huge, where a lot of things happen that we can't actually see. But as a scientist, you know about them and forget to mention them. Describing a picture to a blind person will improve your communication skills.

Another excellent way to start is to find a group already working on accessibility and collaborate with them. You can join the "Astronomía Inclusiva" group (Spanish), even for

${ }^{21} \mathrm{https}: / /$ santalucia.cl/ 
working on non-astronomy subjects, or contact IAU Working Group on Astronomy for Equity and Inclusion, ${ }^{22}$ where you can find resources, publications and good practices.

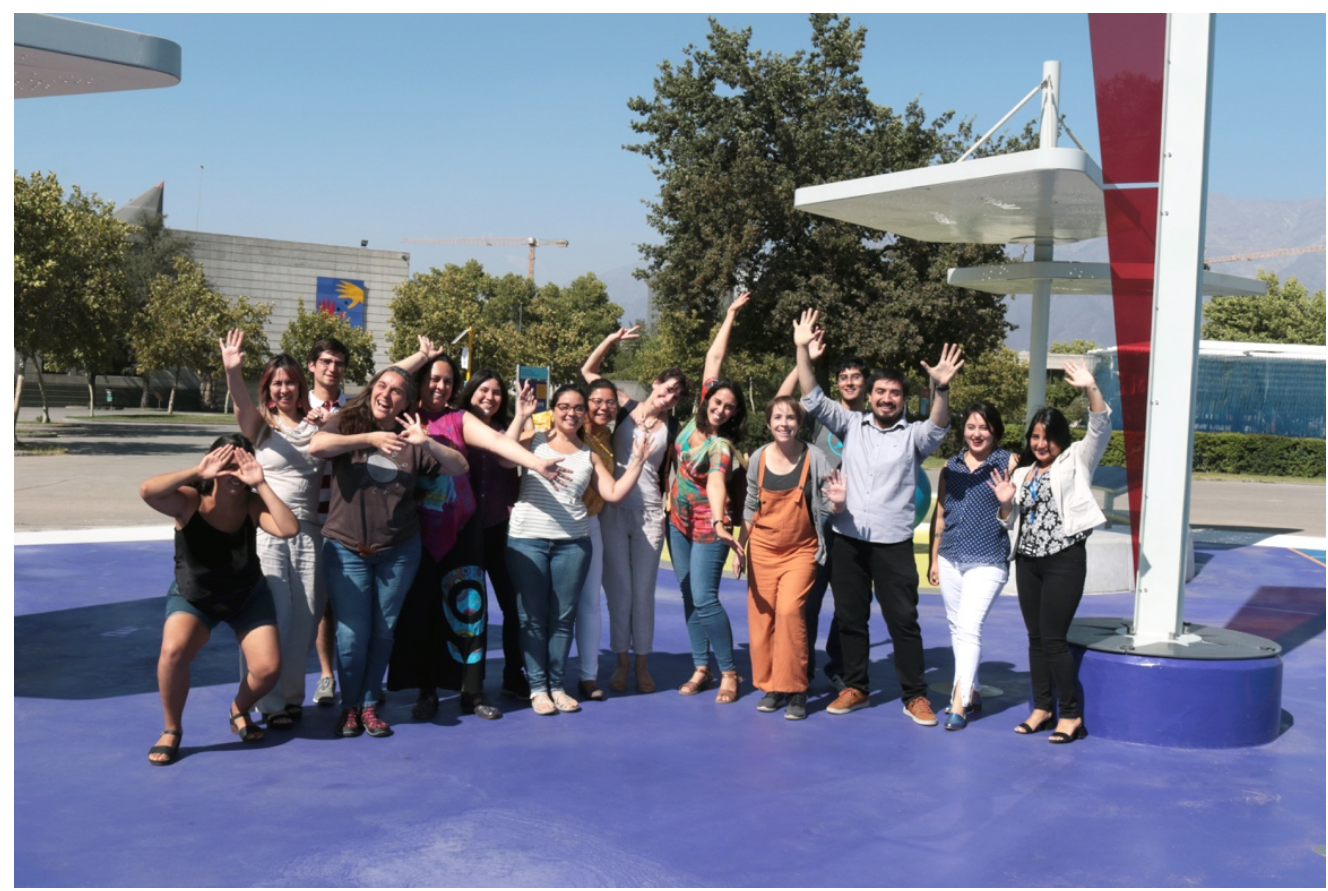

Figure 6: “Astronomía Inclusiva” group meeting at MIM museum. January 2020

\section{Find us in social media:}

Núcleo de Astronomía UDP: @astroudp at Instagram ${ }^{23}$, Twitter ${ }^{24}$ and Facebook ${ }^{25}$.

“Astronomía Inclusiva” Group: @astro.inclusiva at Instagram ${ }^{26}$, Twitter ${ }^{27}$ and Facebook ${ }^{28}$.

\section{References}

[1] Diaz Merced, Wanda L. (2013) Sound for the exploration of space physics data. PhD thesis, University of Glasgow.

[2] Convention on the Rights of Persons with Disabilities. United Nations. Retrieved 7 July 2021.

[3] Kramer, Gregory, ed. (1994). Auditory Display: Sonification, Audification, and Auditory Interfaces. Santa Fe Institute Studies in the Sciences of Complexity. Proceedings Volume XVIII. Reading, MA: Addison-Wesley. ISBN 978-0-201-62603-2

\footnotetext{
$22 \mathrm{https} / / /$ iau.org/science/scientific bodies/working groups/259

${ }^{23} \mathrm{https} / / / \mathrm{www}$. instagram.com/astroudp/

${ }^{24} \mathrm{https}: / /$ twitter.com/AstroUDP

${ }^{25} \mathrm{https}: / /$ www.facebook.com/difusionastroudp

${ }^{26} \mathrm{https}: / / \mathrm{www}$. instagram.com/astro.inclusiva/

${ }^{27} \mathrm{https}: / /$ twitter.com/astro_inclusiva

${ }^{28} \mathrm{https}$ //www.facebook.com/astroinclusiva
} 